\title{
CORRIGENDUM
}

\section{Electromagnetic zonal flow residual responses - Corrigendum}

\author{
Peter J. Catto ${ }^{\oplus}$, Felix I. Parra and István Pusztai \\ doi:10.1017/S0022377817000472, Published by Cambridge University Press,
}

Regrettably there are several errors in our paper. We present the corrections for the Appendix first as they are the source of some of the errors in the main body of the paper.

\section{Appendix A. Endless integrals}

The second term in the $\kappa \rightarrow 1$ limit of $\overline{\cos \theta}$ in (A 50) should be to the minus one power with a prefactor of $1 / 4$ rather than $1 / 2$.

The sign should be positive in the last form of (A 53) and both forms of (A 54).

Following (A 59), add the remark that 'The $\vartheta$-independent contribution of order $\varepsilon$ is neglected'.

Equation (A 61) should be

$$
\begin{aligned}
\varsigma & =\frac{30 \sqrt{2}}{\pi}\left\{\int_{0}^{1} \frac{\mathrm{d} \kappa \kappa[2 E(\kappa)-K(\kappa)]^{2}}{(1-\varepsilon+2 \varepsilon \kappa)^{7 / 2} K(\kappa)}+\int_{0}^{1} \frac{\mathrm{d} k k\left[2 E(k)-\left(2-k^{2}\right) K(k)\right]^{2}}{\left[(1-\varepsilon) k^{2}+2 \varepsilon\right]^{7 / 2} K(k)}\right\} \\
& \simeq \frac{30 \sqrt{2}}{\pi}\left\{\int_{0}^{1} \frac{\mathrm{d} \kappa \kappa}{K(\kappa)}[2 E(\kappa)-K(\kappa)]^{2}+\int_{0}^{1} \frac{\mathrm{d} k}{k^{6} K(k)}\left[2 E(k)-\left(2-k^{2}\right) K(k)\right]^{2}\right\} \\
& \simeq 4.363 .
\end{aligned}
$$

Equations (A 65) and (A 66) should read

$$
\left(M^{2} \underline{B}_{0}^{2} / 2 n T^{2} B_{0}^{2}\right) \int \mathrm{d}^{3} v f_{0} v_{\perp}^{2}\left(v_{\|}^{2}-\overline{v_{\|}^{2}}\right) \simeq\left(4 \varepsilon-\varsigma \varepsilon^{3 / 2}\right) \cos \theta+\cdots
$$

and

$$
\left(M^{2} \underline{B}_{0}^{2} / 2 n T^{2} B_{0}^{2}\right) \int \mathrm{d}^{3} v f_{0} v_{\perp}^{2}\left[v_{\|} \overline{v_{\|}}-\left(v_{\|}^{2}+\overline{v_{\|}^{2}}\right) / 2\right] \simeq-5 \gamma \varepsilon^{3 / 2} / 2-\left(\varsigma \varepsilon^{3 / 2} / 2\right) \cos \vartheta+\cdots .
$$

Equation (A 71) must be multiplied by 2 , so that $\chi \simeq 0.2262$.

A more precise evaluation of the $\cos \vartheta$ coefficient in (A 80) replaces $v=5 \gamma \simeq 8.2$ by $v \equiv \pi^{-1} \varepsilon^{-5 / 2} \oint \mathrm{d} \theta I \cos \vartheta=7.96$.

This change affects (5.15), (5.19)-(5.21), (5.31), (5.43), (5.45), (6.2), and (6.5)-(6.7).

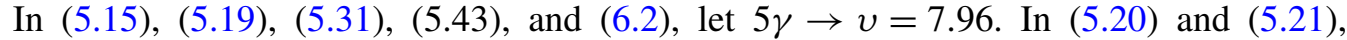
make the replacement $1-\varepsilon^{1 / 2} / 2 \gamma q^{2} \rightarrow[(v-3 \gamma) / 2 \gamma]\left[1-(v-4 \gamma) \varepsilon^{1 / 2} /(v-3 \gamma) q^{2}\right]$.

$\dagger$ Email address for correspondence: catto@psfc.mit.edu 
In (6.5) and (6.6), the same replacement gives final forms with $1 \rightarrow(v-3 \gamma) / 2 \gamma$. In (5.45), let $5 \gamma \tilde{A}_{\|}^{(0)} \rightarrow v \tilde{A}_{\|}^{(0)}$ and $5 \gamma \tilde{\Phi}^{(0)} \rightarrow[v(v-3 \gamma) / 2 \gamma] \tilde{\Phi}^{(0)}$, while in (6.2) let $5 \gamma \rightarrow v(v-3 \gamma) / 2 \gamma$.

\section{Kinetic equation and solution}

Two lines above $(3.8), \Delta_{s}$ should be $\Delta_{s}^{\prime}$, so the result reads $B_{p} / B_{t}=(\varepsilon / q)[1-$ $\left.\Delta_{s}^{\prime} \cos \vartheta+O\left(\varepsilon^{2}\right)\right]$.

\section{Quasineutrality and Ampere's law}

Remove the last sentence of the paragraph ending after (5.9).

The left side of (5.10) and the beginning of the paragraph including it should read as follows. Expanding for $Q=k_{\perp} v_{\|} / \Omega_{p} \ll 1$, inserting $H$, and neglecting $\varepsilon$ corrections to $k_{\perp}^{2} \rho_{i}^{2}$ terms, but retaining all $Q^{2}, \beta$, and $\varepsilon^{3 / 2}$ corrections to the $\tilde{B}_{\|}$and $\tilde{B}_{\|}^{(0)}$ terms in (5.5), recalling $\tilde{\Phi}=\langle\tilde{\Phi}\rangle$, and using unperturbed quasineutrality, the perpendicular Ampere's law becomes

$$
\begin{aligned}
\tilde{B}_{\|} & +\Sigma \frac{\pi M^{2}}{T B_{0}^{2}} \int \mathrm{d}^{3} v f_{0} v_{\perp}^{4} \overline{\tilde{B}_{\|}}-\tilde{B}_{\|}^{(0)}\left\{\frac{\underline{B}_{0}}{B_{0}}-\varepsilon \Sigma \frac{4 \pi M}{\beta B_{0}^{2}} \int \mathrm{d}^{3} v f_{0} v_{\perp}^{2} \overline{\cos \vartheta}\right. \\
& \left.+\Sigma \frac{4 \pi M}{\beta B_{0}^{3}} \int \mathrm{d}^{3} v f_{0} v_{\perp}^{2}\left[Q \overline{Q B_{0}}-\frac{1}{2}\left(Q^{2} \overline{B_{0}}+\overline{Q^{2} B_{0}}\right)\right]\right\} \simeq \text { right side. }
\end{aligned}
$$

In the $\tilde{B}_{\|}^{(0)} / \beta$ term of $(5.11)$, the factor $\overline{v_{\|}}-v_{\|}$should be replaced by $\overline{v_{\|}}-B_{0}^{-1} v_{\|} \overline{B_{0}}$ and in the last term we use $\int \mathrm{d}^{3} v f_{0} v_{\|}(Q-\bar{Q})\left(M v^{2} / 2 T-3 / 2\right)=\int \mathrm{d}^{3} v f_{0} v_{\|}(Q-\bar{Q})$.

Correct the ' $a$ ' term on the right side of (5.12) by the replacement $3 \varepsilon a / 2 \rightarrow 3 a / 2$ and then fix the left side of (5.12) by keeping $\beta_{i}$ and $\beta$ corrections to read

$$
(1+\beta)\left(\left\langle\tilde{B}_{\|}\right\rangle-\tilde{B}_{\|}^{(0)}\right)+\frac{5 \gamma \beta_{i}}{4 \beta} \varepsilon^{3 / 2} k_{\perp}^{2} \rho_{p i}^{2} \tilde{B}_{\|}^{(0)} \simeq \text { corrected right side. }
$$

Remove the last sentence of the paragraph that ends after (5.12).

In (5.13), remove the $a \Delta_{s}^{\prime}$ term on the left side and on the right remove the term proportional to $\beta_{i} \varepsilon\left(\langle\tilde{\Phi}\rangle-\tilde{\Phi}^{(0)}\right)$ and other $\langle\tilde{\Phi}\rangle$ and $\tilde{\Phi}^{(0)}$ terms that are negligible in what follows, to obtain

$$
\begin{aligned}
& \left\langle\tilde{A}_{\|}\right\rangle\left[1+\frac{\omega_{p}^{2}}{k_{\perp}^{2} c^{2}}\left(1-\gamma \varepsilon^{3 / 2}\right)\right]-\tilde{A}_{\|}^{(0)}\left[\left(1+\frac{\omega_{p}^{2}}{k_{\perp}^{2} c^{2}}\right)\left(1-\gamma \varepsilon^{3 / 2}\right)\right] \\
& \simeq a \frac{\varepsilon \omega_{p}^{2}}{2 k_{\perp}^{2} c^{2}}\left(1-\gamma \varepsilon^{3 / 2}\right)+\frac{\mathrm{i} \gamma q \varepsilon^{1 / 2}}{k_{\perp}} \tilde{B}_{\|}^{(0)}+\mathrm{i} \frac{c \sigma}{4 v_{i}} \beta_{i} q^{3} k_{\perp} \rho_{i} \varepsilon^{-1 / 2}\langle\tilde{\Phi}\rangle .
\end{aligned}
$$

In (5.14), make the replacements $a \chi \varepsilon^{3 / 2} \rightarrow a \chi \varepsilon^{1 / 2}$ and $\langle\tilde{\Phi}\rangle \rightarrow\langle\tilde{\Phi}\rangle / 2$ and fix the $\tilde{B}_{\|}^{(0)}$ and ' $b$ ' terms to read

$$
\begin{gathered}
\left(1+\frac{\varsigma \beta \varepsilon^{1 / 2}}{4}\right) b \simeq \varepsilon \tilde{B}_{\|}^{(0)}\left(1-\frac{\varsigma}{5} \varepsilon^{1 / 2}-\frac{\varsigma \beta_{i}}{4 \beta} \varepsilon^{1 / 2} k_{\perp}^{2} \rho_{p i}^{2}\right)+\frac{c \varsigma \beta_{i} q^{2} k_{\perp}^{2} \rho_{i}}{4 \varepsilon^{1 / 2} v_{i}}\langle\tilde{\Phi}\rangle \\
-\frac{\mathrm{i} q \beta k_{\perp}}{2}\left(3-\varsigma \varepsilon^{1 / 2}\right)\left[\left\langle\tilde{A}_{\|}\right\rangle-\tilde{A}_{\|}^{(0)}\left(1+\frac{k_{\perp}^{2} c^{2}}{\omega_{p}^{2}}\right)\right]-\frac{\mathrm{i} \chi \varepsilon^{1 / 2} q \beta k_{\perp} a}{4} .
\end{gathered}
$$

Following (5.14), correct the constants to read $\chi \simeq 0.226$ and $\varsigma \simeq 4.36$. 
Correcting (5.15), because $k_{\perp}^{2}$ in (4.11) must be a flux function, whereas on the left side of (5.4) $k_{\perp}^{2} \rightarrow k_{\perp}^{2}\left(1+2 \Delta_{s}^{\prime} \cos \vartheta\right)$, and multiplying the $\tilde{B}_{\|}^{(0)}$ term by 2 gives

$$
\begin{aligned}
& a\left[1+\frac{\varepsilon\left(\varepsilon+2 \Delta_{s}^{\prime}\right) \omega_{p}^{2}}{2 k_{\perp}^{2} c^{2}}\right] \simeq\left(\varepsilon+2 \Delta_{s}^{\prime}\right)\left[\frac{\omega_{p}^{2}}{k_{\perp}^{2} c^{2}}\left\langle\tilde{A}_{\|}\right\rangle-\left(\frac{\omega_{p}^{2}}{k_{\perp}^{2} c^{2}}+1\right) \tilde{A}_{\|}^{(0)}\right] \\
& +\frac{\mathrm{i} 2 q}{k_{\perp}} \tilde{B}_{\|}^{(0)}+\frac{\mathrm{i} c}{4 v_{i}} \beta_{i} q k_{\perp} \rho_{i}\left[\left(5 \gamma q^{2} \varepsilon^{-1 / 2}+4\right)\langle\tilde{\Phi}\rangle-3 \tilde{\Phi}^{(0)}\right] .
\end{aligned}
$$

Equations (5.16) and (5.17) are the same as (5.15) and (5.13), but with the $\tilde{B}_{\|}^{(0)}$ terms removed.

The large skin depth limit of (5.16) and (5.17) are

$$
\frac{v_{i}}{c}\left\langle\tilde{A}_{\|}\right\rangle \rightarrow \frac{v_{i}}{c} \tilde{A}_{\|}^{(0)}\left(1-\gamma \varepsilon^{3 / 2}\right)+\mathrm{i} \frac{\sigma \beta_{i}}{4 \varepsilon^{1 / 2}} q^{3} k_{\perp} \rho_{i}\langle\tilde{\Phi}\rangle
$$

and

$$
\frac{v_{i}}{c} a \rightarrow-\frac{v_{i}}{c}\left(\varepsilon+2 \Delta_{s}^{\prime}\right) \tilde{A}_{\|}^{(0)}+\mathrm{i} \frac{\beta_{i}}{4} q k_{\perp} \rho_{i}\left[\left(5 \gamma q^{2} \varepsilon^{-1 / 2}+4\right)\langle\tilde{\Phi}\rangle-3 \tilde{\Phi}^{(0)}\right] .
$$

The small skin depth limits $\left(k_{\perp}^{2} c^{2} / \omega_{p}^{2} \ll 1\right)$ of (5.16) and (5.17), (5.20) and (5.21), should read

$$
\frac{v_{i}}{c}\left\langle\tilde{A}_{\|}\right\rangle \simeq \frac{v_{i}}{c}\left[1-\frac{1}{2} \varepsilon\left(\varepsilon+2 \Delta_{s}^{\prime}\right)\right] \tilde{A}_{\|}^{(0)}+\mathrm{i} \frac{1}{4} \beta_{i} \varepsilon^{2} k_{\perp} \rho_{p i}\left(1-\frac{\varepsilon^{1 / 2}}{2 \gamma q^{2}}\right) \tilde{\Phi}^{(0)}
$$

and

$$
\frac{v_{i}}{c} a \simeq-\frac{v_{i}}{c}\left(\varepsilon+2 \Delta_{s}^{\prime}\right) \tilde{A}_{\|}^{(0)}+\mathrm{i} \frac{1}{2} \beta_{i} \varepsilon k_{\perp} \rho_{p i}\left(1-\frac{\varepsilon^{1 / 2}}{2 \gamma q^{2}}\right) \tilde{\Phi}^{(0)},
$$

upon using (5.9) to calculate the response to $\tilde{\Phi}^{(0)}$, because the corrections to $\langle\tilde{\Phi}\rangle$ due to $\tilde{A}_{\|}^{(0)}$ are negligible (as will be found from quasineutrality shortly). Notice that at small skin depth the relation $\left\langle\tilde{A}_{\|}\right\rangle \simeq \tilde{A}_{\|}^{(0)}+\varepsilon a / 2$ holds. Keeping $\varepsilon^{1 / 2}$ corrections, we note that for $\tilde{A}_{\|}^{(0)}=0$ stronger poloidal variation occurs, as $\left\langle\tilde{A}_{\|}\right\rangle / a \simeq \varepsilon / 2$.

Correcting the full parallel Ampere's law without inserting (5.9), but otherwise making the changes already noted, (5.22) and (5.23) are the same as the corrected (5.15) and (5.13).

Evaluating (5.12) and (5.14) without inserting (5.9), (5.24) and (5.25) should read as follows:

$$
\begin{aligned}
& (1+\beta)\left(\left\langle\tilde{B}_{\|}\right\rangle-\tilde{B}_{\|}^{(0)}\right)+\frac{5 \gamma \beta_{i}}{4 \beta} \varepsilon^{3 / 2} k_{\perp}^{2} \rho_{p i}^{2} \tilde{B}_{\|}^{(0)} \simeq \frac{c \beta_{i} k_{\perp}^{2} \rho_{i}}{4 v_{i}}\left[\left(5 \gamma q^{2} \varepsilon^{-1 / 2}+3\right)\langle\tilde{\Phi}\rangle-3 \tilde{\Phi}^{(0)}\right] \\
& +\frac{\mathrm{i} q k_{\perp} \beta}{4}\left\{5 \gamma \varepsilon^{1 / 2}\left[\left\langle\tilde{A}_{\|}\right\rangle-\tilde{A}_{\|}^{(0)}\left(1+\frac{k_{\perp}^{2} c^{2}}{\omega_{p}^{2}}\right)\right]+3 a\right\}
\end{aligned}
$$

and

$$
\begin{aligned}
& \left(1+\frac{\varsigma \beta \varepsilon^{1 / 2}}{4}\right) b \simeq \varepsilon \tilde{B}_{\|}^{(0)}\left(1-\frac{\varsigma}{5} \varepsilon^{1 / 2}-\frac{\varsigma \beta_{i}}{4 \beta} \varepsilon^{1 / 2} k_{\perp}^{2} \rho_{p i}^{2}\right) \\
& -\frac{\mathrm{i} q \beta k_{\perp}}{2}\left(3-\varsigma \varepsilon^{1 / 2}\right)\left[\left\langle\tilde{A}_{\|}\right\rangle-\tilde{A}_{\|}^{(0)}\left(1+\frac{k_{\perp}^{2} c^{2}}{\omega_{p}^{2}}\right)\right]-\frac{\mathrm{i} \chi q \beta \varepsilon^{1 / 2} k_{\perp} a}{4}+\frac{c \varsigma q^{2} \beta_{i} k_{\perp}^{2} \rho_{i}}{4 v_{i} \varepsilon^{1 / 2}}\langle\tilde{\Phi}\rangle,
\end{aligned}
$$


where all $\langle\tilde{\Phi}\rangle$ and $\tilde{\Phi}^{(0)}$ terms are corrected. The line following (5.25) and (5.26) should be deleted and replaced by the following sentence. Equations (5.22) and (5.23) are only consistent with

$$
\left\langle\tilde{A}_{\|}\right\rangle \simeq \tilde{A}_{\|}^{(0)}+\frac{\varepsilon}{2} a
$$

in the small skin depth limit, for which field lines switch between irrational and rational when $q_{1}=i k_{\perp} B_{p}^{-1} \tilde{A}_{\|}^{(0)} \neq 0$.

When $\tilde{B}_{\|}^{(0)}=0$, the $\omega_{p}^{2} / k_{\perp}^{2} c^{2} \rightarrow 0$ limits of (5.24) and (5.25) correct (5.27) and (5.28) to read

$$
\frac{v_{i}\left\langle\tilde{B}_{\|}\right\rangle}{k_{\perp} c} \rightarrow \frac{\beta_{i} k_{\perp} \rho_{i}}{4}\left[\left(5 \gamma q^{2} \varepsilon^{-1 / 2}+3\right)\langle\tilde{\Phi}\rangle-3 \tilde{\Phi}^{(0)}\right]-\frac{\mathrm{i} 5 \gamma q \beta \varepsilon^{1 / 2} k_{\perp}^{2} c^{2}}{4 \omega_{p}^{2}} \frac{v_{i}}{c} \tilde{A}_{\|}^{(0)}
$$

and

$$
\frac{v_{i} b}{k_{\perp} c} \rightarrow \frac{\mathrm{i} q \beta k_{\perp}^{2} c^{2}}{2 \omega_{p}^{2}}\left(3-\varsigma \varepsilon^{1 / 2}\right) \frac{v_{i}}{c} \tilde{A}_{\|}^{(0)}+\frac{\varsigma q^{2} \beta_{i} k_{\perp} \rho_{i}}{4 \varepsilon^{1 / 2}}\langle\tilde{\Phi}\rangle .
$$

The sentences that follow should then read as follows. Consequently, when $\tilde{\Phi}^{(0)}=0$, we see that the ratio of the $\tilde{A}_{\|}^{(0)}$ terms gives $b /\left\langle\tilde{B}_{\|}\right\rangle \simeq-6 / 5 \gamma \varepsilon^{1 / 2}$, so rather strong poloidal variation occurs in $\tilde{B}_{\|}$. For $\tilde{A}_{\|}^{(0)}=0$, the poloidal variation of $\tilde{B}_{\|}$is strong since $b /\left\langle\tilde{B}_{\|}\right\rangle \simeq \varsigma / 2 \gamma \simeq 1.3$.

For $\tilde{B}_{\|}^{(0)}=0$ and $\beta \ll 1$, the small skin depth $\left(\omega_{p}^{2} / k_{\perp}^{2} c^{2} \gg 1\right)$ limits of (5.24) and (5.25) yield the corrected (5.29) and (5.30) to be

$$
\frac{v_{i}\left\langle\tilde{B}_{\|}\right\rangle}{k_{\perp} c} \simeq \frac{\beta_{i} k_{\perp} \rho_{i}}{4}\left[\left(5 \gamma q^{2} \varepsilon^{-1 / 2}+3\right)\langle\tilde{\Phi}\rangle-3 \tilde{\Phi}^{(0)}\right]-\frac{\mathrm{i} 3\left(\varepsilon+2 \Delta_{s}^{\prime}\right) q \beta}{4} \frac{v_{i}}{c} \tilde{A}_{\|}^{(0)}
$$

and

$$
\frac{v_{i} b}{k_{\perp} c} \simeq \frac{\mathrm{i} q \beta \varepsilon^{1 / 2}\left(\varepsilon+2 \Delta_{s}^{\prime}\right)}{4}\left[\chi+\left(3-\varsigma \varepsilon^{1 / 2}\right) \varepsilon^{1 / 2}\right] \frac{v_{i}}{c} \tilde{A}_{\|}^{(0)}+\frac{\varsigma q^{2} \beta_{i} k_{\perp} \rho_{i}}{4 \varepsilon^{1 / 2}}\langle\tilde{\Phi}\rangle,
$$

where we assume $\varepsilon^{3 / 2} \gg k_{\perp}^{2} c^{2} / \omega_{p}^{2} \gg \Delta_{s}^{\prime} \varepsilon^{5 / 2}$. The three sentences that follow should be replaced by the following two sentences. When $\tilde{A}_{\|}^{(0)}=0$, the poloidal variation of $\tilde{B}_{\|}$is strong with $b /\left\langle\tilde{B}_{\|}\right\rangle \simeq \varsigma / 2 \gamma \simeq 1.33$. For $\tilde{\Phi}^{(0)}=0$, it is also rather strong, since $b /\left\langle\tilde{B}_{\|}\right\rangle \simeq \chi \varepsilon^{1 / 2} / 3$.

The sentence including (5.31)-(5.32) should be corrected to read as follows. Keeping $\langle\tilde{\Phi}\rangle$ terms, the $\omega_{p}^{2} / k_{\perp}^{2} c^{2} \rightarrow 0$ limit gives

$$
k_{\perp} a \rightarrow \mathrm{i} 2 q \tilde{B}_{\|}^{(0)}+\mathrm{i} \frac{5 \gamma}{4} \varepsilon^{1 / 2} q^{2} k_{\perp} \rho_{p i} \frac{c \beta_{i} k_{\perp}\langle\tilde{\Phi}\rangle}{v_{i}}
$$

and

$$
k_{\perp}\left\langle\tilde{A}_{\|}\right\rangle \rightarrow \mathrm{i} \gamma q \varepsilon^{1 / 2} \tilde{B}_{\|}^{(0)}+\mathrm{i} \frac{\sigma}{4} \varepsilon^{1 / 2} q^{2} k_{\perp} \rho_{p i} \frac{c \beta_{i} k_{\perp}\langle\tilde{\Phi}\rangle}{v_{i}} .
$$

Evaluating (5.12) and (5.14) without inserting (5.9), (5.33) and (5.34) should read as follows:

$$
\begin{aligned}
\left\langle\tilde{B}_{\|}\right\rangle & \rightarrow\left[1-\frac{3}{2} q^{2} \beta-\frac{5 \gamma \beta_{i}}{4 \beta} \varepsilon^{3 / 2} k_{\perp}^{2} \rho_{p i}^{2}\right] \tilde{B}_{\|}^{(0)}+\frac{5 \gamma}{4} q \varepsilon^{1 / 2} k_{\perp} \rho_{p i} \frac{c \beta_{i} k_{\perp}\langle\tilde{\Phi}\rangle}{v_{i}} \\
\quad= & {\left[1-\frac{3}{2} q^{2} \beta\right] \tilde{B}_{\|}^{(0)} }
\end{aligned}
$$


and

$$
\begin{gathered}
\left(1+\frac{\varsigma \beta \varepsilon^{1 / 2}}{4}\right) b \rightarrow\left[\varepsilon\left(1-\frac{\varsigma}{5} \varepsilon^{1 / 2}-\frac{\varsigma \beta_{i}}{4 \beta} \varepsilon^{1 / 2} k_{\perp}^{2} \rho_{p i}^{2}\right)+\frac{\chi+3 \gamma}{2} q^{2} \beta \varepsilon^{1 / 2}\right] \tilde{B}_{\|}^{(0)} \\
+\frac{\varsigma}{4} q \varepsilon^{1 / 2} k_{\perp} \rho_{p i} \frac{c \beta_{i} k_{\perp}\langle\tilde{\Phi}\rangle}{v_{i}}=\left[\varepsilon\left(1-\frac{\varsigma}{5} \varepsilon^{1 / 2}\right)+\frac{\chi+3 \gamma}{2} q^{2} \beta \varepsilon^{1 / 2}\right] \tilde{B}_{\|}^{(0)}
\end{gathered}
$$

where $c \beta k_{\perp}\langle\tilde{\Phi}\rangle / v_{i} \tilde{B}_{\|}^{(0)} \simeq k_{\perp} \rho_{i}$ is inserted as this result will be determined from quasineutrality in the next subsection, and the $\beta$ correction on the left side is small in the final form.

Correcting the next paragraph, it should read as follows. In the small skin depth limit $\varepsilon^{3 / 2} \omega_{p}^{2} / k_{\perp}^{2} c^{2} \gtrsim 1 \gg \Delta_{s}^{\prime} \varepsilon^{5 / 2} \omega_{p}^{2} / k_{\perp}^{2} c^{2}$ for finite $\beta \ll 1$ and $\tilde{\Phi}^{(0)}=0=\tilde{A}_{\|}^{(0)}$, we use (5.22) and (5.23) along with the quasineutrality result $c \beta k_{\perp}\langle\tilde{\Phi}\rangle / v_{i} \tilde{B}_{\|}^{(0)} \simeq k_{\perp} \rho_{i}$ to find $\varepsilon a \simeq 2\left\langle\tilde{A}_{\|}\right\rangle$. Then (5.22)-(5.25) give

$$
\begin{gathered}
k_{\perp} a \simeq \mathrm{i} 2 q \tilde{B}_{\|}^{(0)}, \\
k_{\perp}\left\langle\tilde{A}_{\|}\right\rangle \simeq \mathrm{i} q \varepsilon \tilde{B}_{\|}^{(0)}, \\
\left\langle\tilde{B}_{\|}\right\rangle \simeq \tilde{B}_{\|}^{(0)}\left(1-\frac{3}{2} q^{2} \beta\right)
\end{gathered}
$$

and

$$
b \simeq \tilde{B}_{\|}^{(0)}\left[\varepsilon\left(1-\frac{\varsigma}{5} \varepsilon^{1 / 2}\right)+\frac{\chi}{2} q^{2} \varepsilon^{1 / 2} \beta\right]
$$

for $\beta \sim \varepsilon k_{\perp}^{2} \rho_{p i}^{2}$. As a result, for this $\tilde{B}_{\|}^{(0)} \neq 0$ case with $\tilde{\Phi}^{(0)}=0$ and $\tilde{A}_{\|}^{(0)}=0$, the poloidal variation of $\tilde{A}_{\|}$is strong with $\left\langle\tilde{A}_{\|}\right\rangle / a \simeq \varepsilon / 2$, while the poloidal variation of $\tilde{B}_{\|}$is weak with $b /\left\langle\tilde{B}_{\|}\right\rangle \simeq \varepsilon$.

In $\S 5.4$, there are sign errors in the $\tilde{A}_{\|}$terms of $(5.41)-(5.45)$. The signs can be corrected by changing the signs of all $\tilde{A}_{\|}, \tilde{A}_{\|}^{(0)},\left\langle\tilde{A}_{\|}\right\rangle$, and ' $a$ ' terms. In addition, $5 \gamma / 16$ replaces $(\sigma+5 \gamma) / 16$ in $(5.45)$ and $\varepsilon \rightarrow \varepsilon+2 \Delta_{s}^{\prime}$ in the $\tilde{A}_{\|}^{(0)}$ term, so it becomes

$$
\begin{aligned}
\left(\frac{\gamma q^{2}}{\varepsilon^{1 / 2}}+1\right)\langle\tilde{\Phi}\rangle= & \tilde{\Phi}^{(0)}\left[1+\frac{5 \gamma}{16} q^{2} \beta_{i} \varepsilon^{3 / 2} k_{\perp}^{2} \rho_{p i}^{2}\right]-\mathrm{i} \frac{5 \gamma v_{i}}{8 c} q^{2} \varepsilon^{1 / 2}\left(\varepsilon+2 \Delta_{s}^{\prime}\right) k_{\perp} \rho_{p i} \tilde{A}_{\|}^{(0)} \\
& +\frac{\gamma v_{i}}{c \beta} q \varepsilon^{1 / 2} \rho_{p i} \tilde{B}_{\|}^{(0)}
\end{aligned}
$$

\section{Zonal flow responses in terms of initial field values: 15 test cases}

Section 6 summarizes the small skin depth zonal flow residual responses for the various initial perturbations. The complete summary of the corrected results is as follows.

$$
\begin{gathered}
\text { 6.1. } A_{\|}^{(0)}=0=B_{\|}^{(0)} \\
\left(\frac{\gamma q^{2}}{\varepsilon^{1 / 2}}+1\right) \frac{\langle\tilde{\Phi}\rangle}{\tilde{\Phi}^{(0)}}=1+\frac{5 \gamma}{16} q^{2} \beta_{i} \varepsilon^{3 / 2} k_{\perp}^{2} \rho_{p i}^{2} \\
\frac{v_{i}\left\langle\tilde{B}_{\|}\right\rangle}{k_{\perp} c \tilde{\Phi}^{(0)}}=\frac{\beta_{i} \varepsilon k_{\perp} \rho_{p i}}{2 q}
\end{gathered}
$$




$$
\begin{aligned}
& \frac{v_{i} b}{k_{\perp} c \tilde{\Phi}^{(0)}}=\frac{\varsigma \varepsilon \beta_{i} k_{\perp} \rho_{p i}}{4 \gamma q} \\
& \frac{v_{i}\left\langle\tilde{A}_{\|}\right\rangle}{\mathrm{i} c \tilde{\Phi}^{(0)}}=\frac{1}{4} \varepsilon^{2} \beta_{i} k_{\perp} \rho_{p i}\left(1-\frac{\varepsilon^{1 / 2}}{2 \gamma q^{2}}\right) \simeq \frac{1}{4} \varepsilon^{2} \beta_{i} k_{\perp} \rho_{p i}, \\
& \frac{v_{i} a}{\mathrm{i} c \tilde{\Phi}^{(0)}}=\frac{1}{2} \varepsilon \beta_{i} k_{\perp} \rho_{p i}\left(1-\frac{\varepsilon^{1 / 2}}{2 \gamma q^{2}}\right) \simeq \frac{1}{2} \varepsilon \beta_{i} k_{\perp} \rho_{p i} . \\
& \text { 6.2. } \Phi^{(0)}=0=B_{\|}^{(0)} \\
& \frac{\mathrm{i} c\langle\tilde{\Phi}\rangle}{v_{i} \tilde{A}_{\|}^{(0)}}=\frac{5 \varepsilon\left(\varepsilon+2 \Delta_{s}^{\prime}\right) k_{\perp} \rho_{p i}}{8\left(1+\varepsilon^{1 / 2} / \gamma q^{2}\right)} \simeq \frac{5}{8} \varepsilon\left(\varepsilon+2 \Delta_{s}^{\prime}\right) k_{\perp} \rho_{p i}, \\
& \frac{\left\langle\tilde{A}_{\|}\right\rangle}{\tilde{A}_{\|}^{(0)}}=1-\frac{1}{2} \varepsilon\left(\varepsilon+2 \Delta_{s}^{\prime}\right), \\
& \frac{a}{\tilde{A}_{\|}^{(0)}}=-\left(\varepsilon+2 \Delta_{s}^{\prime}\right), \\
& \frac{\mathrm{i}\left\langle\tilde{B}_{\|}\right\rangle}{k_{\perp} \tilde{A}_{\|}^{(0)}}=\frac{3}{4} q \beta\left(\varepsilon+2 \Delta_{s}^{\prime}\right), \\
& \frac{b}{\mathrm{i} k_{\perp} \tilde{A}_{\|}^{(0)}}=\frac{\chi}{4} q \beta \varepsilon^{1 / 2}\left(\varepsilon+2 \Delta_{s}^{\prime}\right) \text {. } \\
& \text { 6.3. } \Phi^{(0)}=0=A_{\|}^{(0)} \\
& \frac{c \beta k_{\perp}\langle\tilde{\Phi}\rangle}{v_{i} \tilde{B}_{\|}^{(0)}}=\frac{\varepsilon k_{\perp} \rho_{p i}}{q\left(1+\varepsilon^{1 / 2} / \gamma q^{2}\right)} \simeq \frac{\varepsilon k_{\perp} \rho_{p i}}{q}, \\
& \frac{k_{\perp}\left\langle\tilde{A}_{\|}\right\rangle}{\mathrm{i} \tilde{B}_{\|}^{(0)}}=q \varepsilon, \\
& \frac{k_{\perp} a}{\mathrm{i} \tilde{B}_{\|}^{(0)}}=2 q, \\
& \frac{\left\langle\tilde{B}_{\|}\right\rangle}{\tilde{B}_{\|}^{(0)}}=1-\frac{3}{2} q^{2} \beta, \\
& \frac{b}{\tilde{B}_{\|}^{(0)}}=\varepsilon\left(1-\frac{\varsigma}{5} \varepsilon^{1 / 2}\right)+\frac{\chi}{2} q^{2} \varepsilon^{1 / 2} \beta .
\end{aligned}
$$

All small skin depth results are consistent with $\left\langle\tilde{A}_{\|}\right\rangle \simeq \tilde{A}_{\|}^{(0)}+\varepsilon a / 2$, but field lines can only switch between irrational and rational when $q_{1}=\mathrm{i} k_{\perp} B_{p}^{-1} \tilde{A}_{\|}^{(0)} \neq 0$.

\section{Conclusions}

The last sentence of the third paragraph from the end should read as follows. The poloidally varying response is larger than the flux surface averaged response for $\tilde{A}_{\|}$ and comparable for $\tilde{B}_{\|}$. 
In the first sentence of the second paragraph from the end the word 'also' should not appear. Replace the last sentence in the same paragraph with the following sentence. However, the poloidal variation of $\tilde{B}_{\|}$is almost comparable to its flux surface averaged value.

\section{Acknowledgements}

P.J.C. was supported by the US Department of Energy, grant DE-FG02-91ER-54109. I.P. was supported by an International Career grant (Dnr. 330-2014-631) from Vetenskapsrådet, and the Marie Sklodowska Curie Actions, Cofund, Project INCA 600398. One benefit of having to write this Corrigendum is that it gives us another (and, hopefully, final) chance to thank (and curse) A. Schekochihin of Oxford University for suggesting this problem in March 2013 at a workshop at the Wolfgang Pauli Institute in Vienna, Austria. 